\title{
Mitigation of Transceiver Bandwidth Limitations using Multi-Subcarrier Signals
}

\author{
Fernando P. Guiomar ${ }^{1}$, Luca Bertignono ${ }^{1}$, Antonino Nespola ${ }^{2}$ and \\ Andrea Carena ${ }^{1}$ \\ ${ }^{1}$ DET, Politecnico di Torino, Corso Duca degli Abruzzi, 24, 10129, Torino, Italy \\ ${ }^{2}$ Istituto Superiore Mario Boella, via Pier Carlo Boggio 61, 10138 Torino, Italy \\ e-mail: fernando.guiomar@polito.it
}

\begin{abstract}
In this paper we demonstrate a low-complexity pre-emphasis technique for electronic subcarriermultiplexed signals to mitigate transceiver bandwidth limitations. As opposed to traditional FFT/FIR-based preemphasis techniques, we propose a simple and effective power-ratio optimization between electronic subcarriers that requires only 1 real multiplication per subcarrier.
\end{abstract}

Keywords: coherent optical communications, subcarrier multiplexing, pre-emphasis

\section{INTRODUCTION}

Digital pre-emphasis is a well-known signal processing technique that is now ubiquitous in coherent optical communication systems, with the aim to counteract the electrical bandwidth limitations of coherent transceivers [1]. This approach is generally implemented through a static fractionally-spaced finite-impulse response (FIR) or fast-Fourier transform (FFT) based filter, operating at the sampling rate of the digital-to-analog converter (DAC) [2], [3], [4]. These techniques have been proposed for single-carrier Nyquist-shaped transmission systems with high symbol-rates exceeding 32 GBaud and high-order modulation formats. As an alternative to singlecarrier transceivers, electronic subcarrier-multiplexing has been recently attracting the attention of the optical communications community due to its inherent advantages in term of robustness to chromatic dispersion [5] and nonlinear impairments [6], [7]. While the standard pre-emphasis filtering techniques can also be applied to electrical subcarrier-multiplexing signals by processing all subcarriers as a single entity, new opportunities arise on the design of pre-emphasis techniques that treat each subcarrier independently. In particular, digital preemphasis can be optimized to overcome a widely reported issue associated with electrical subcarrier-multiplexed signals: the varying signal-to-noise ratio (SNR) per subcarrier, caused by the frequency-dependent transceiver response [8].

In this paper, we propose a simple and low-complexity ( 1 real multiplication per subcarrier) power-ratio adjustment technique that can replace typical fractionally-spaced pre-emphasis filtering in electrical subcarriermultiplexed systems. Our technique is experimentally demonstrated in a back-to-back laboratorial scenario, in which all subcarriers are operating within $0.2 \mathrm{~dB}$ of implementation penalty variation.

\section{EXPERIMENTAL SETUP AND RESULTS}

The back-to-back (B2B) experimental setup is depicted in Fig. 1. The PM-16QAM transmitted signal is composed of 8 subcarriers modulated at 4 GBaud each (total symbol-rate of 32 GBaud). In this work, no digital pre-emphasis techniques based on FFT/FIR filtering are considered. The digital signal is loaded in a $64 \mathrm{GSa} / \mathrm{s}$ DAC with $\sim 8 \mathrm{GHz}$ electrical bandwidth and an effective number of bits (ENOB) of approximately 5.5. The four electrical analog outputs generated by the DAC (in-phase and quadrature components on both polarizations) are amplified by wideband amplifiers. The optical carrier is generated by an external cavity laser (ECL), which feeds a dual-polarization Mach-Zehnder modulator (DP-MZM), where the DAC electrical outputs are optically modulated to generate the subcarrier-multiplexed PM-16QAM signal. The optical signal-to-noise ratio (OSNR) is set by using an optical noise source controlled by variable optical attenuator (VOA). A second ECL is used as a local oscillator for the coherent receiver, where the received optical signal is downconverted to the

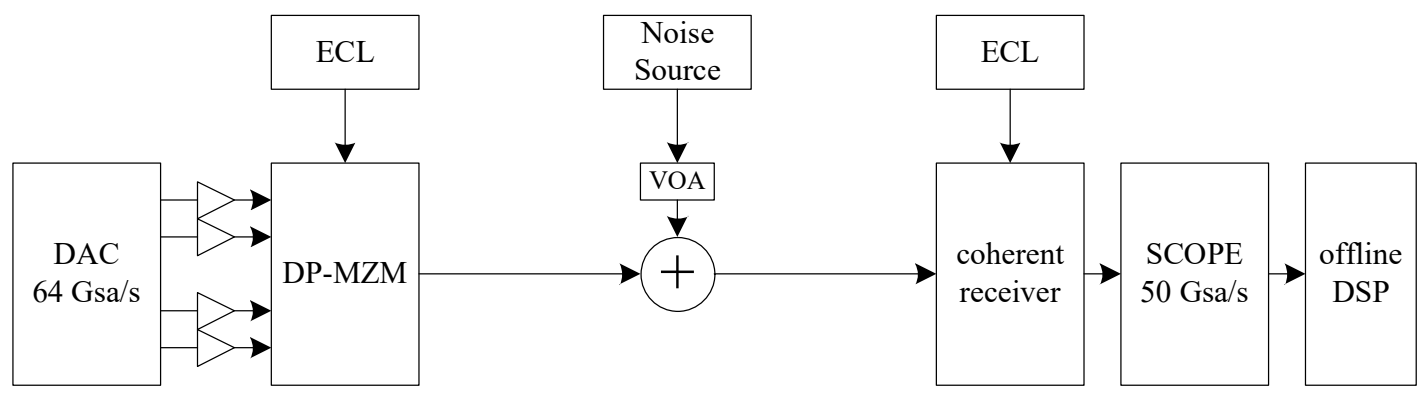

Figure 1: Experimental setup for the B2B performance assessment. 


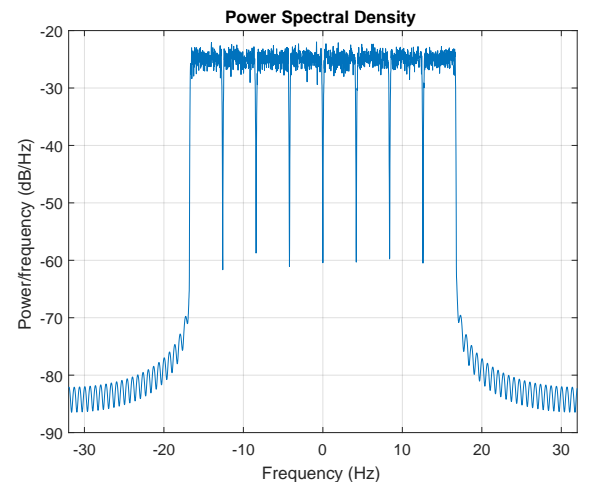

(a)

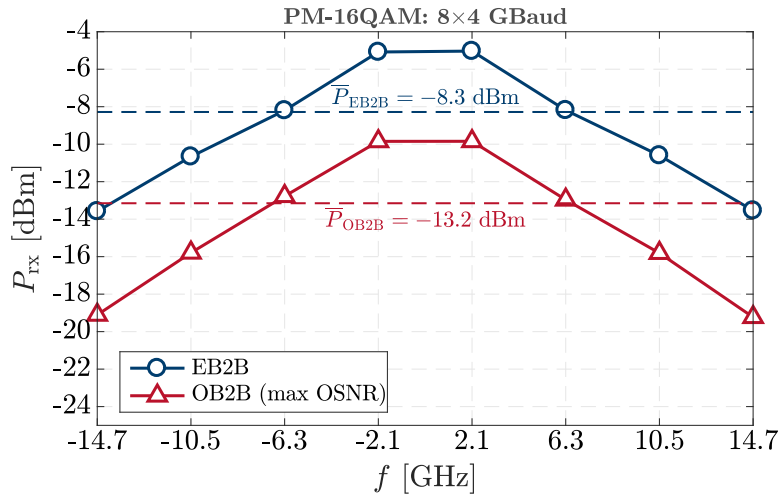

(c)

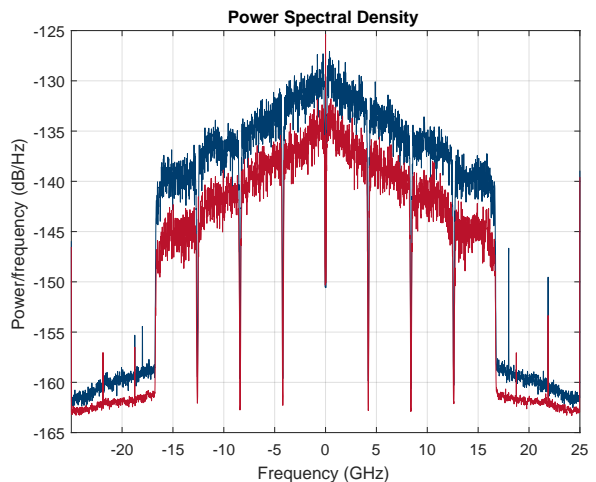

(b)

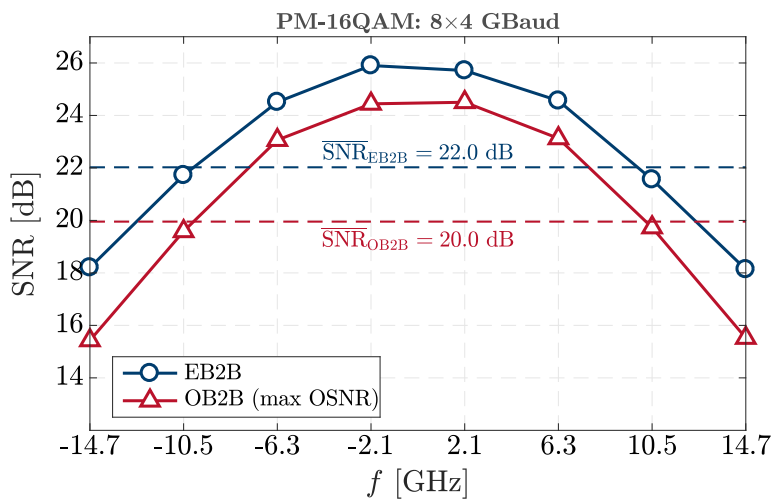

(d)

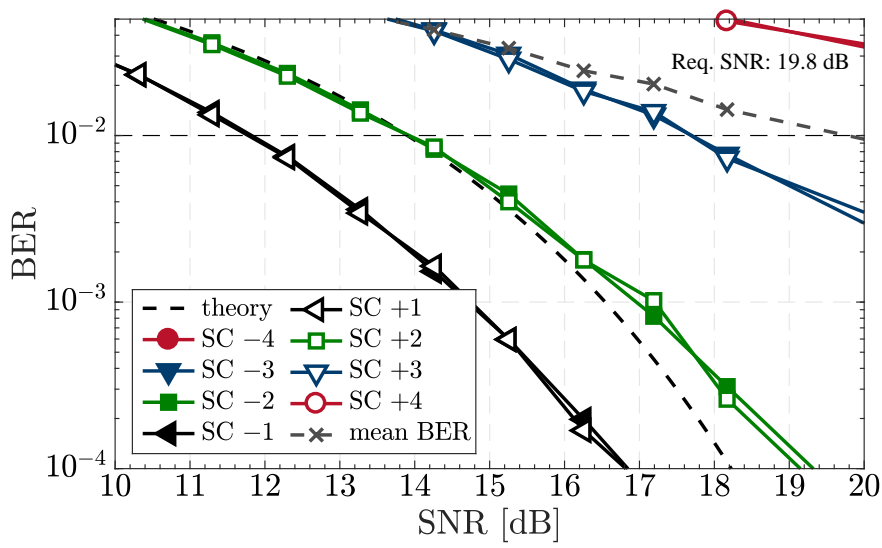

(e)

Figure 2: Experiment without any pre-emphasis applied: a flat-power spectrum is generated at the output of the DAC. a) Electrical spectrum at the DAC output; b) Received spectrum: electrical (blue) and optical (red); c) received power per subcarrier; d) measured SNR per subcarrier at maximum OSNR (no noise loading); e) B2B performance characterization.

electrical domain and decomposed into its quadrature components. Finally, the electrical outputs of the coherent receiver are digitally sampled by a real-time digital oscilloscope with $50 \mathrm{Gsa} / \mathrm{s}$ and $\sim 30 \mathrm{GHz}$ of bandwidth. Digital signal processing follows, employing standard DSP subsystems for polarization demultiplexing, linear filtering and carrier recovery. The system performance is assessed through the counted bit-error-rate (BER) or the measured signal-to-noise ratio (SNR) of the signal constellation.

We start our analysis conducting an experiment without applying any pre-emphasis technique: we set a flat-power spectrum at the output of the DAC, thereby emulating the worst case scenario, in which no countermeasures are taken against the transceiver bandwidth limitations. The spectrum of the digital signal uploaded in the DAC is shown in Fig. 2a, whereas the received signal spectrum (after the oscilloscope) is shown in Fig. 2b, both in electrical (blue) and optical (red) B2B configuration. The aggressive low-pass filtering incurred by the transceiver is well visible, with about $10 \mathrm{~dB}$ loss between the central and edge subcarriers, as can also be 


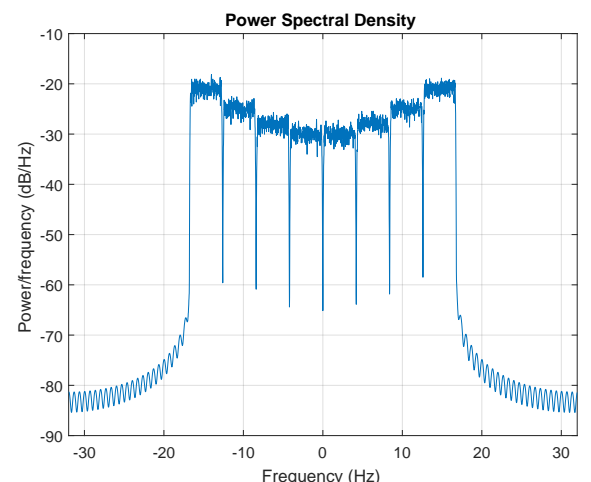

(a)

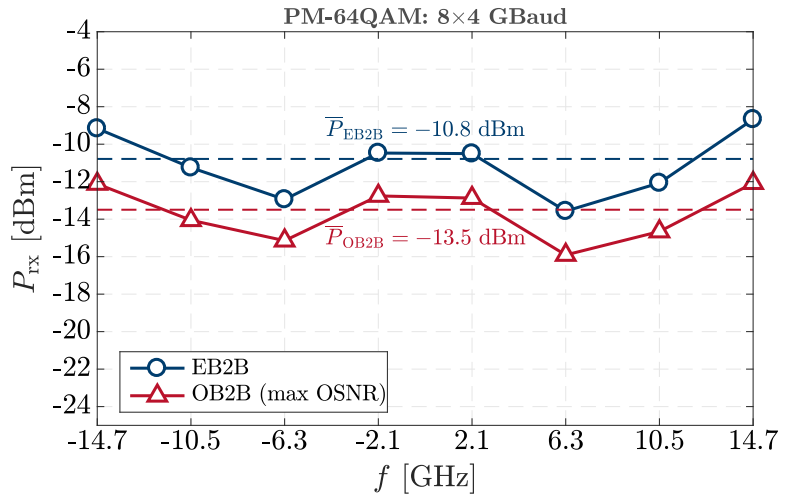

(c)

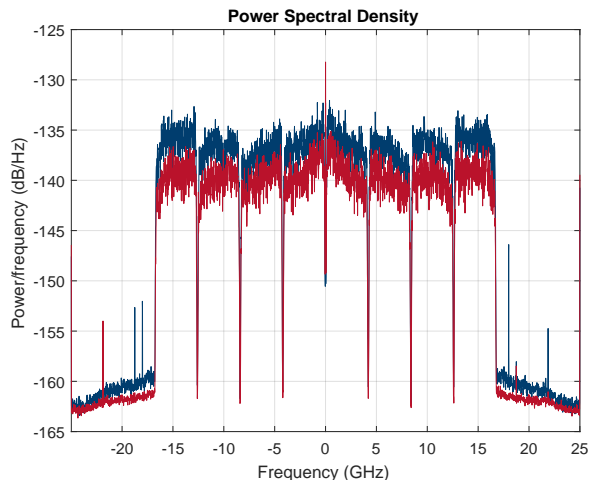

(b)

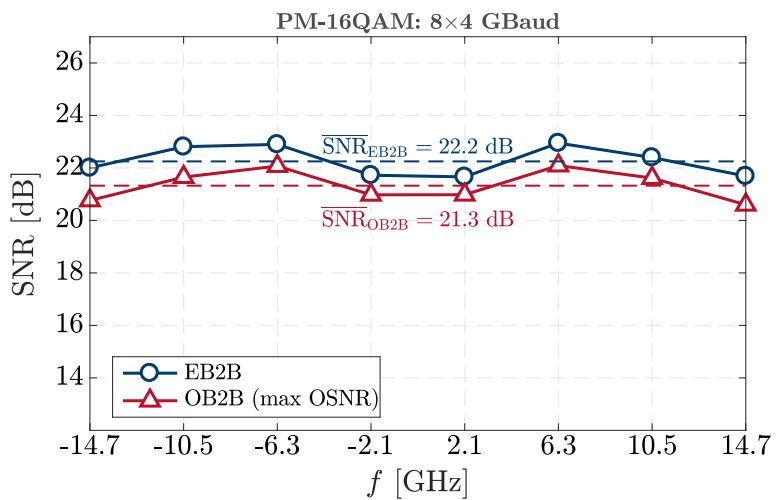

(d)

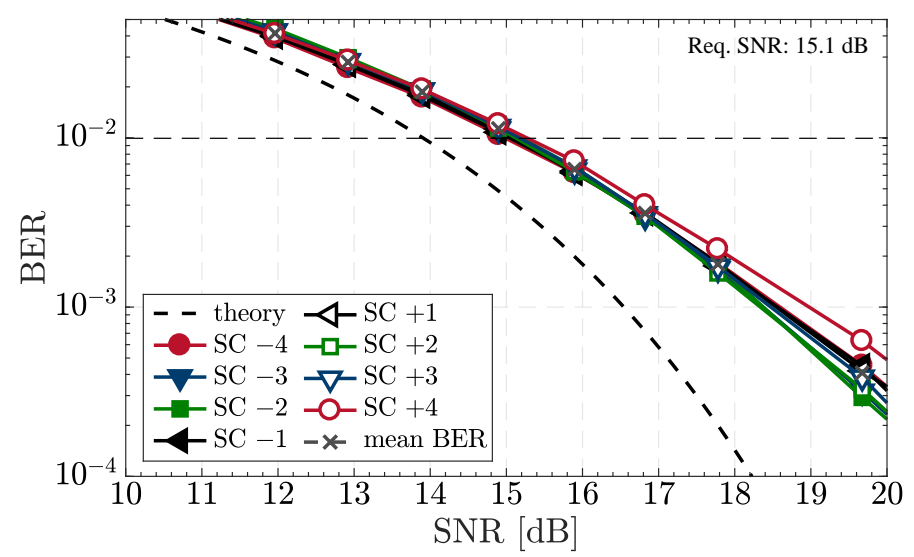

(e)

Figure 3: Experiment applying the proposed pre-emphasis technique. a) Electrical spectrum at the DAC output; b) Received spectrum: electrical (blue) and optical (red); c) received power per subcarrier; d) measured SNR per subcarrier at maximum OSNR (no noise loading); e) B2B performance characterization.

confirmed in Fig. 2c that shows the measured received power per subcarrier. This causes a steep degradation of SNR (measured over the received constellation) towards the edge subcarriers, as can be seen in Fig. 2d, which is approximately proportional to the loss of power. Consequently, the required SNR per subcarrier to achieve a given target BER (in this work we have established a target BER of $10^{-2}$ ) is also substantially different. Indeed, in Fig. 2e we can see the performances are grouped in pairs of symmetric subcarriers (around the baseband). The subcarriers near to the baseband are the ones with lowest required SNR, while the ones in the edges require approximately $>10 \mathrm{~dB}$ higher SNR. For this scenario, the required SNR corresponding to the average BER among all subcarriers was found to be $19.8 \mathrm{~dB}$, corresponding to approximately $6 \mathrm{~dB}$ of penalty against the theory $(\sim 14 \mathrm{~dB})$.

Then, we proceeded to optimize the power-ratio between subcarriers, so that their performances are equalized.

The obtained power-ratios, defined as the ratio between the power of each subcarrier and the lowest power among all subcarriers, were $P R=[8.43 ; 4.72 ; 1.55 ; 0 ;-0.05 ; 1.51 ; 4.57 ; 8.16] \mathrm{dB}$. The spectrum of the corre- 
sponding transmitted digital signal is shown in Fig. 3a, whereas the received signal spectrum is shown in Fig. $3 b$. Similarly to a standard pre-emphasis technique based on digital filtering, the transmitted spectrum becomes shaped in such a way that approximately inverts the low-pass profile of the transceiver. The major advantage of our approach is that only a simple real multiplication is required per subcarrier, in contrast with the $O\left(N_{\text {taps }}^{2}\right)$ complexity required by FIR filtering (where $N_{\text {taps }}$ is the number of FIR taps) or the $O\left(N_{\mathrm{FFT}} \log _{2}\left(N_{\mathrm{FFT}}\right)\right)$ complexity required by FFT-based filtering (where $N_{\mathrm{FFT}}$ is the FFT block-size). As can be observed in Fig. 3b, all subcarrier are now at similar power levels. Since only a single power-ratio factor is assigned to each subcarrier, the consequence is that each of the received subcarriers is not exactly flat in power, showing some spectrum tilt due to the low-pass of the transceiver. Nevertheless, this tilt is very narrow in frequency (symbol-rate of each subcarrier) and can be easily compensated at the received by the adaptive linear equalizer. As shown in Fig. 3e, the BER versus SNR B2B characterization now exhibits very similar performance between subcarriers (within

$0.2 \mathrm{~dB}$ of required SNR). Consequently, also the average performance of the entire subcarrier-multiplexing signal is significantly improved, now yielding a required SNR of $15.1 \mathrm{~dB}$ to work at the target BER of $10^{-2}$, only $\sim 1.1 \mathrm{~dB}$ away from the theory. It is also worth noting that, although the transmitted power-ratio per subcarrier has been optimized to minimize the performance gap between subcarriers, the corresponding received power per subcarrier is not perfectly flat, as can be seen in Fig. 3c. In fact, a residual received power-ratio of 2-3 $\mathrm{dB}$ is required to equalize the subcarrier performances, which is due to the presence of non-white frequencydependent noise, generated both by the DAC (mostly at high frequencies, due to the reduction of ENOB) and by the wideband amplifiers (mostly at low-frequencies). This clearly shows that equalization of performances between subcarriers in subcarrier-multiplexed signals cannot follow standard FIR/FFT techniques, which take only into account the equalization of the received power. In contrast, our technique adaptively equalize the performance of all subcarriers, at the cost of a simple real multiplication per subcarrier.

\section{CONCLUSIONS}

We have experimentally demonstrated a novel low-complexity pre-emphasis technique for subcarrier multiplexed signals that relies on the power-ratio adjustment between subcarriers in order to counteract the low-pass filtering imposed by the transceiver. The proposed technique requires only a single real multiplication per subcarrier and allows for the adaptive equalization of the subcarrier-multiplexed signal performance. In a B2B experimental setup, we have demonstrated the operation of our technique over a 32 GBaud PM-16QAM signal composed of 8 subcarriers, yielding only $\sim 1.1 \mathrm{~dB}$ of implementation penalty at a target BER of $10^{-2}$.

\section{ACKNOWLEDGMENTS}

This work was supported by the European Commission through a Marie Skodowska-Curie individual fellowship, project Flex-ON (653412).

\section{REFERENCES}

[1] D. Rafique, A. Napoli, S. Calabro and B. Spinnler: Digital preemphasis in optical communication systems: On the DAC Requirements for Terabit Transmission Applications, Opt. Express, vol. 32, no. 19, pp. 32473256, 2014.

[2] J. Wang, C. Xie and Z. Pan: Optimization of DSP to generate spectrally efficient 16QAM Nyquist-WDM signals, IEEE Photonics Technol. Lett., vol. 25, no. 8, pp. 772-775, 2013.

[3] J. Zhang, H. C. Chien, Y. Xia, Y. Chen and J. Xiao: A novel adaptive digital pre-equalization scheme for bandwidth limited optical coherent system with DAC for signal generation, in Proc. Optical Fiber Conference, paper W3K.4, 2014.

[4] A. Napoli, M. M. Mezghanni, T. Rahman, D. Rafique, R. Palmer, B. Spinnler, S. Calabrò, C. Castro, M. Kuschnerov and M. Bohn: Digital compensation of bandwidth limitations for high-speed DACs and ADCs, J. Lightwave Technol., vol. 34, no. 13, pp. 3053-3064, 2016.

[5] M. Malekiha, I. Tselniker and D. V. Plant, Chromatic dispersion mitigation in long-haul fiber-optic communication networks by sub-band partitioning, Opt. Express, vol. 23, no. 25, pp. 32654-32663, 2015.

[6] P. Poggiolini, A. Nespola, Y. Jiang, G. Bosco, A. Carena, L. Bertignono, S. M. Bilal, S. Abrate and F. Forghieri: Analytical and experimental results on system maximum reach increase through symbol rate optimization, J. Lightwave Technol., vol. 34, no. 8, pp. 1872-1885, 2016.

[7] F. P. Guiomar, A. Carena, G. Bosco, L. Bertignono, A. Nespola and P. Poggiolini: Nonlinear mitigation on subcarrier-multiplexed PM-16QAM optical systems, Opt. Express, vol. 25, no. 4, pp. 4298-4311, 2017.

[8] T. A. Eriksson, F. Buchali, W. Idler, L. Schmalen and G. Charlet: Electronically subcarrier multiplexed PM-32QAM with optimized FEC overheads, in Proc. Optical Fiber Conference, paper W3J.4, 2017. 